\title{
Recurrent Urinary System Carcinoma
}

National Cancer Institute

\section{Source}

National Cancer Institute. Recurrent Urinary System Carcinoma. NCI Thesaurus. Code

C7623.

Reemergence of a urinary system carcinoma after a period of remission. 